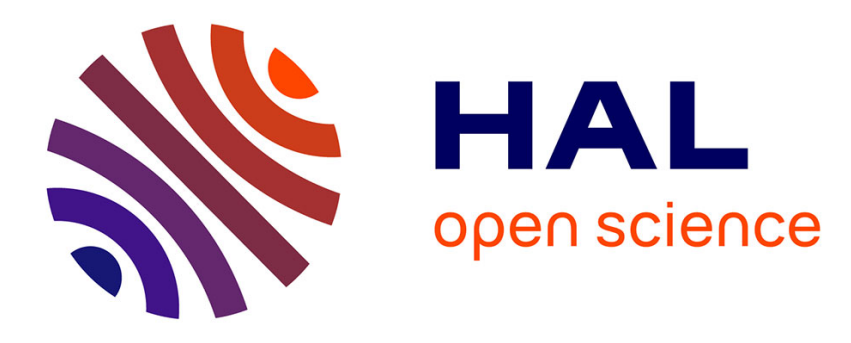

\title{
Reconstructing Shapes with Guarantees by Unions of Convex Sets
}

\author{
Dominique Attali, André Lieutier
}

\section{To cite this version:}

Dominique Attali, André Lieutier. Reconstructing Shapes with Guarantees by Unions of Convex Sets. SoCG 2010 - 26th Annual Symposium on Computational Geometry, Jun 2010, Snowbird, Utah, United States. pp.344-353, 10.1145/1810959.1811015 . hal-00427035v3

\section{HAL Id: hal-00427035 \\ https://hal.science/hal-00427035v3}

Submitted on 31 Mar 2010

HAL is a multi-disciplinary open access archive for the deposit and dissemination of scientific research documents, whether they are published or not. The documents may come from teaching and research institutions in France or abroad, or from public or private research centers.
L'archive ouverte pluridisciplinaire HAL, est destinée au dépôt et à la diffusion de documents scientifiques de niveau recherche, publiés ou non, émanant des établissements d'enseignement et de recherche français ou étrangers, des laboratoires publics ou privés. 


\title{
Reconstructing Shapes with Guarantees by Unions of Convex Sets*
}

\author{
[Extended Abstract] ${ }^{\dagger}$ \\ Dominique Attali \\ Gipsa-lab, Grenoble, France \\ Dominique.Attali@grenoble-inp.fr \\ André Lieutier \\ Aix-en-Provence, France \\ Andre.Lieutier@wanadoo.fr
}

\begin{abstract}
A simple way to reconstruct a shape $A \subset \mathbb{R}^{N}$ from a sample $P$ is to output an $r$-offset $P+r B$, where $B=\left\{x \in \mathbb{R}^{N} \mid\right.$ $\|x\| \leq 1\}$ designates the unit Euclidean ball centered at the origin. Recently, it has been proved that the output $P+r B$ is homotopy equivalent to the shape $A$, for a dense enough sample $P$ of $A$ and for a suitable value of the parameter $r$ 12,22 . In this paper, we extend this result and find convex sets $C \subset \mathbb{R}^{N}$, besides the unit Euclidean ball $B$, for which $P+r C$ reconstructs the topology of $A$. This class of convex sets includes in particular $N$-dimensional cubes in $\mathbb{R}^{N}$. We proceed in two steps. First, we establish the result when $P$ is an $\varepsilon$-offset of $A$. Building on this first result, we then consider the case when $P$ is a finite noisy sample of $A$.
\end{abstract}

\section{Categories and Subject Descriptors}

F.2.2 [Analysis of Algorithms and Problem Complexity]: Nonnumerical Algorithms and Problems - Geometrical problems and computations, Computations on discrete structures; I.3.5 [Computer Graphics]: Computational Geometry and Object Modeling

\section{General Terms}

Theory, Algorithms

\section{Keywords}

Shape reconstruction, offsets, Minkowski sum, union of convex sets, sampling, homotopy equivalence

\footnotetext{
${ }^{*}$ This work is partially supported by ANR Project GIGA ANR-09-BLAN-0331-01.

${ }^{\dagger}$ A full version of this paper is available at
} http://hal.archives-ouvertes.fr/hal-00427035

Permission to make digital or hard copies of all or part of this work for personal or classroom use is granted without fee provided that copies are not made or distributed for profit or commercial advantage and that copies bear this notice and the full citation on the first page. To copy otherwise, to republish, to post on servers or to redistribute to lists, requires prior specific permission and/or a fee.

SCG'10, June 13-16, 2010, Snowbird, Utah, USA.

Copyright 2010 ACM 978-1-4503-0016-2/10/06 ...\$10.00.

\section{INTRODUCTION}

In this paper, we study the Minkowski sum between a convex set and a point set that samples a shape, generalizing previous results that establish the Minkowski sum retrieves the topology of the shape when the convex set is a Euclidean ball.

\section{Prior work and problem.}

Motivated by surface reconstruction from 3D scan data and manifold learning from point clouds, several authors have formulated precise conditions under which a reconstruction algorithm outputs a topologically correct approximation of a shape, given as input a possibly noisy sample of it $4,14,10,22,12$. Maybe one of the simplest algorithm consists in outputting a Euclidean $r$-offset of the sample, that is the union of Euclidean balls with radius $r$ centered on the sample points. Assuming the reach of the shape is positive and data points form a sufficiently dense and accurate sample of the shape, authors in 22,12 have established that $r$-offsets of the data points are homotopy equivalent to the shape for suitable values of the offset parameter $r$ (see Figure 1. left). The aim of this work is to understand how this result generalizes when, instead of unions of Euclidean balls, we consider for the reconstruction unions of translated and scaled copies of a convex set $C$ centered on the data points. In other words, writing $B$ for the unit Euclidean ball centered at the origin and letting $P$ be a sample of the shape $A$, we would like to understand what happens if we replace the Euclidean $r$-offset $P+r B$ by the Minkowski sum $P+r C$. Do we keep the topology of the shape $A$ as in Figure 1 middle or do we lose it as in Figure 1 right? We are particularly interested in the case where $C$ is a polytope.

\section{Motivation.}

Our motivation to study this question is two-fold. First, in many practical applications such as stereo vision or image analysis, the accuracy of measures varies in magnitude according to the direction of measurements. In this context, it seems reasonable to recover the topology of the shape, using a convex set which takes into account the anisotropy of the measurement device. Second, we believe that unions of cubes could present some advantages over unions of Euclidean balls for topological computations in high dimension. In practice, the reconstruction represented by an $\alpha$-offset is replaced by the more convenient corresponding $\alpha$-shape which shares the same homotopy type 15$]$. Indeed, the $\alpha$ shape has a simpler geometry and, being a simplicial complex, can benefit from existing theorems and algorithms ded- 

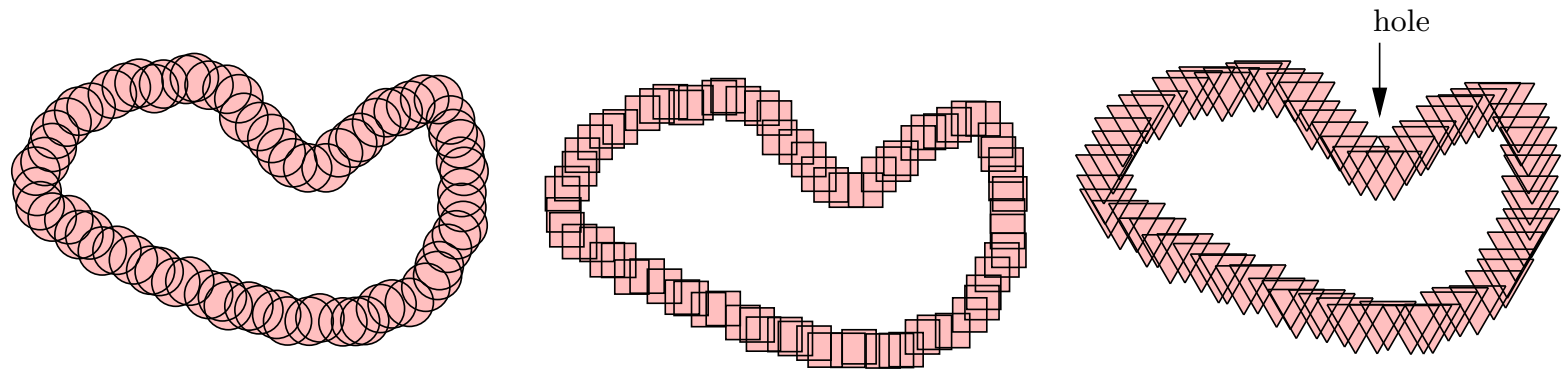

Figure 1: The union of disks and squares retrieve the topology of the sampled curve unlike the union of triangles.

icated to topological computations. However, if the ambient dimension is large, the $\alpha$-shape may have a high complexity $[1]$ and its computation may be rather expensive and requires sophisticated data structures 8]. Our idea to circumvent this problem is the following. Given $\varepsilon>0$, we define the cubical grid $G_{\varepsilon}=(\varepsilon \mathbb{Z})^{N} \subset \mathbb{R}^{N}$ and replace the sample $P \subset \mathbb{R}^{N}$ by a nearby sample $P_{\varepsilon} \subset G_{\varepsilon}$. Applying our result to the unit $N$-dimensional cube $C=[-1,1]^{N} \in \mathbb{R}^{N}$, we shall see that the set $P_{\varepsilon}+k \varepsilon C$ retrieves the homotopy type of the shape, for some well-chosen integer $k$. Hence, our result allows us to reconstruct with the right homotopy type a shape by a union of voxels with vertices the cubical grid (see 5] for a precise statement of this corollary and [6] for an application). Such a "cubical set" has a simpler structure than the $\alpha$-shape and may be more convenient for topological computations in high dimension. Following this idea, our work might contribute to build a bridge between the point of view of distance functions in computational topology and the world of voxels in digital image processing.

\section{Chosen approach and contributions.}

A first idea to tackle the problem mentioned above is to use the framework of semi-concave functions. Specifically, one can associate to any symmetric convex set $C$ with a nonempty interior a norm $\|.\|_{C}$ defined by $x \mapsto\|x\|_{C}=\inf \{\alpha>$ $0 \mid x \in \alpha C\}$. The balls of the associated distance $d_{C}$ are translated and scaled copies of $C$. The metric $d_{C}$ is invariant by translation but is not isotropic unless $C$ is the Euclidean ball. Suppose the boundary of $C$ is smooth with a bounded curvature. Given a subset $Y \subset \mathbb{R}^{N}$, the squared distanceto- $Y$ function $x \mapsto \min _{y \in Y}\|x-y\|_{C}^{2}$ is semi-concave [9] and has therefore a generalized gradient which induces a continuous flow. Hence, a theory similar to what has been done in the Euclidean case 10, 11, 12 can be developed. However, we are interested in convex sets, such as polytopes, whose boundary are not necessarily smooth nor has a bounded curvature. It follows that the semi-concavity property is lost and no generalized gradient of the squared distance-to- $Y$ function can induce a flow. The proof technique used in this paper should be of independent interest since it overcomes the limitation of flow-based methods and applies to convex sets with non-smooth boundary. Taking inspiration in 22 where a deformation retract of a Euclidean offset of the sample onto the shape is constructed explicitly, we move away from this approach and introduce a new proof scheme based a sandwich lemma (Lemma 1). Results in this paper are positive as well as negative. We carefully identify a class of convex sets to which the above result can be extended. We also give examples of convex sets outside this class which won't provide a correct reconstruction. We proceed in two steps. First, we state a reconstruction theorem, when the sample $P$ is an arbitrarily small Euclidean offset of the shape and secondly when $P$ is a finite sample.

\section{Outline.}

Section 2 presents definitions and the formal statements of our two reconstruction theorems. Section 3 proves the first reconstruction theorem and Section 4 proves the second. Section 5 concludes the paper.

\section{STATEMENT OF RESULTS}

Before we state our results in Section 2.3 we first introduce the necessary background in topology in Section 2.1 and identify in Section 2.2 classes of convex sets to which our results will apply.

\subsection{Homotopy equivalences}

First, we review some classical definitions in topology that can be found for instance in 18, 21. Two continuous maps $h, k: X \rightarrow Y$ are homotopic and we write $h \simeq k$ if there is a continuous map $F: X \times[0,1] \rightarrow Y$ such that $F(x, 0)=$ $h(x)$ and $F(x, 1)=k(x)$ for all $x \in X$. Let $f: X \rightarrow Y$ and $g: Y \rightarrow X$ be two continuous maps. Suppose that $f \circ g: Y \rightarrow Y$ is homotopic to the identity map of $Y$ and $g \circ f: X \rightarrow X$ is homotopic to the identity map of $X$, i.e. suppose we have $f \circ g \simeq 1_{Y}$ and $g \circ f \simeq 1_{X}$. Then, the maps $f$ and $g$ are called homotopy equivalences, and each is said to be a homotopy inverse of the other. Furthermore, the spaces $X$ and $Y$ are said to have the same homotopy type, which we denote by $X \simeq Y$. We say that a subspace $A$ of $X$ is a deformation retract of $X$ if there exists a continuous map $H: X \times[0,1] \rightarrow X$ such that $H(x, 0)=x, H(x, 1) \in A$ for all $x \in X$ and $H(a, t)=a$ for all $a \in A$ and all $t \in[0,1]$. Such a function $H$ is called a deformation retraction of $X$ onto $A$. Let $r: X \rightarrow A$ be the retraction defined by $r(x)=$ $H(x, 1)$ and let $i: A \rightarrow X$ the inclusion map. We have $i \circ r \simeq 1_{X}$ and $r \circ i=1_{A}$. Thus, if $A$ is a deformation retract of $X$, the inclusion $i: A \rightarrow X$ is a homotopy equivalence. Note that assuming $X$ deformation retracts to $A$ is stronger than assuming the inclusion map $A \hookrightarrow X$ is a homotopy equivalence, which in turn is stronger than assuming $A \simeq X$, as illustrated in Figure 2. We now state a technical lemma that will provide us key tools in establishing that two shapes have the same topology. 


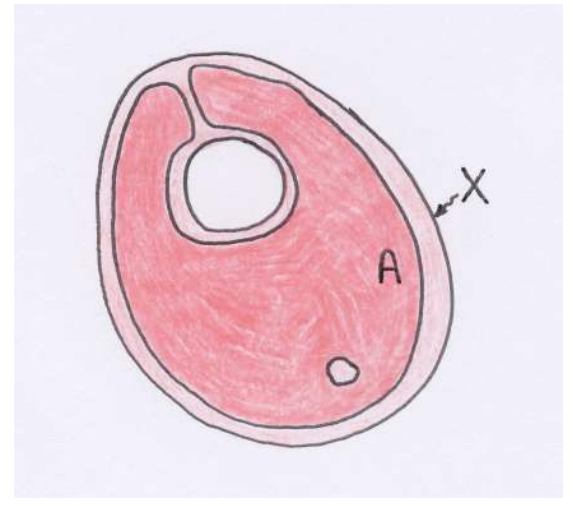

Figure 2: Two nested shapes $A \subset X$ which are close in Hausdorff distance and have the same homotopy type but for which the inclusion $A \hookrightarrow X$ is not a homotopy equivalence.

Lemma 1 (SAndwich Lemma). Consider four nested spaces $A_{0} \subset X_{0} \subset A_{1} \subset X_{1}$. If $A_{1}$ deformation retracts to $A_{0}$ and $X_{1}$ deformation retracts to $X_{0}$, then $X_{0}$ deformation retracts to $A_{0}$. If the inclusions $A_{0} \hookrightarrow A_{1}$ and $X_{0} \hookrightarrow X_{1}$ are homotopy equivalences, then the inclusion $A_{0} \hookrightarrow X_{1}$ is a homotopy equivalence.

Proof. To prove the first part of the lemma, suppose $F$ is a deformation retraction of $A_{1}$ onto $A_{0}$ and $G$ is a deformation retraction of $X_{1}$ onto $X_{0}$. Then, one can check that the map $H: X_{0} \times[0,1] \rightarrow X_{0}$ defined by $H(x, t)=$ $G(F(x, t), 1)$ is a deformation retraction of $X_{0}$ onto $A_{0}$.

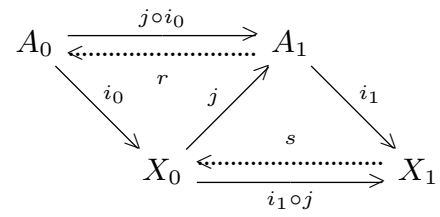

Figure 3: Diagram for the proof of Lemma 1, All arrows but the dotted ones are inclusions.

To prove the second part of the lemma, let $i_{0}: A_{0} \rightarrow X_{0}$, $j: X_{0} \rightarrow A_{1}$ and $i_{1}: A_{1} \rightarrow X_{1}$ denote inclusions (see Figure 3). Suppose $r$ is a homotopy inverse of $j \circ i_{0}$ and $s$ is a homotopy inverse of $i_{1} \circ j$. We prove that the inclusion $k=i_{1} \circ j \circ i_{0}$ from $A_{0}$ to $X_{1}$ is a homotopy equivalence with homotopy inverse $r \circ j \circ s$. Indeed, using the fact that composition preserves the relation $\simeq$, we have $k \circ(r \circ j \circ s)=$ $i_{1} \circ\left(j \circ i_{0} \circ r\right) \circ j \circ s \simeq i_{1} \circ 1_{A_{1}} \circ j \circ s \simeq 1_{X_{1}}$ and similarly $(r \circ j \circ s) \circ k=r \circ j \circ\left(s \circ i_{1} \circ j\right) \circ i_{0} \simeq r \circ j \circ 1_{X_{0}} \circ i_{0} \simeq 1_{A_{0}}$.

\subsection{Properties on convex sets}

In this section, we define two properties that will help us identify classes of convex sets.

\subsection{1 $\quad$-roundness}

We associate to every compact convex set a non-negative real number called the $\theta$-roundness which can be interpreted as a certain kind of curvature. Given a convex set $C$ in $\mathbb{R}^{N}$, the normal cone $\mathcal{N}(x)$ to $C$ at $x$ is the set of unit vectors $n$ such that $(x-y) \cdot n \geq 0$, for all points $y \in C$.
Definition 1. Let $\theta \in[0, \pi]$ and $\varkappa \geq 0$. We say that the compact convex set $C$ is $(\theta, \varkappa)$-round if for all points $c_{1}, c_{2} \in C$ and all vectors $n_{1} \in \mathcal{N}\left(c_{1}\right)$ and $n_{2} \in \mathcal{N}\left(c_{2}\right)$, the following implication holds:

$$
\angle\left(n_{1}, n_{2}\right) \geq \theta \Longrightarrow\left(c_{1}-c_{2}\right) \cdot\left(n_{1}-n_{2}\right) \geq \varkappa\left\|c_{1}-c_{2}\right\|^{2} .
$$

The $\theta$-roundness of $C$ is the supremum of $\varkappa \geq 0$ such that $C$ is $(\theta, \varkappa)$-round.

Note that if the compact convex set $C$ has $\theta$-roundness $\varkappa$, then $C$ is $(\theta, \varkappa)$-round. If $C$ is $(\theta, \varkappa)$-round, then $C$ is $\left(\theta^{\prime}, \varkappa^{\prime}\right)$-round whenever $\theta \leq \theta^{\prime}$ and $\varkappa^{\prime} \leq \varkappa$. It is not difficult to see that $C$ is $(\pi, \varkappa)$-round if and only if the diameter of $C$ is upper bounded by $\frac{2}{\varkappa}$. Suppose the boundary of $C$ is a $\mathrm{C}^{2}$ smooth hypersurface in $\mathbb{R}^{N}$ and orient $\partial C$ such that normals point outside the convex set. Then, for all points $x \in \partial C$, the normal cone at $x$ is reduced to a single vector which is the normal to $\partial C$ at $x$. The absolute values of the principal curvatures at point $x \in \partial C$ are non-negative real numbers $\left|\kappa_{1}(x)\right| \geq\left|\kappa_{2}(x)\right| \ldots \geq\left|\kappa_{N-1}(x)\right|$ and we let $\kappa_{\min }(C)$ be the minimum of $\left|\kappa_{N-1}(x)\right|$ over all points $x \in \partial C$.

LeMma 2. A compact convex set $C$ whose boundary is $\mathrm{C}^{2}$-smooth has 0-roundness $\kappa_{\min }(C)$.

See Appendix $B$ for a proof. For our reconstruction theorems, we shall consider compact convex sets which have the property to be $(\theta, \varkappa)$-round for a positive $\varkappa$ and a small enough $\theta$. Specifically, we will require $\theta \leq \theta_{N}=\arccos \left(-\frac{1}{N}\right)$. Not all convex sets satisfy this property. To construct a counterexample, consider a compact convex set $C$ which is contained in an affine space of dimension $i$ with $0<i<N$. Let $n$ be a unit vector orthogonal to the smallest affine space containing $C$. For every point $c \in C$, both $n$ and its opposite vector $-n$ belong to $\mathcal{N}(c)$. Consider two distinct points $c_{1}, c_{2} \in C$ and let $n_{1}=n$ and $n_{2}=-n$. We have $\angle\left(n_{1}, n_{2}\right)=\pi,\left(c_{1}-c_{2}\right) \cdot\left(n_{1}-n_{2}\right)=0$ and $\left\|c_{1}-c_{2}\right\| \neq 0$, showing that there are no $\varkappa>0$ such that $C$ is $(\pi, \varkappa)$-round. Equivalently, the $\pi$-roundness of $C$ is zero. As a counterexample with a non-empty interior, take a triangular prism in $\mathbb{R}^{3}$. Its $\theta_{3}$-roundness is zero. In the technical report [5], we compute the $\theta_{2}$-roundness of regular polygons in the plane and establish that the $\theta_{N}$-roundness of the $N$-dimensional cube $B_{\infty}=[-1,1]^{N} \subset \mathbb{R}^{N}$ is:

$$
\varkappa\left(B_{\infty}\right)= \begin{cases}\frac{1}{2 \sqrt{2}}\left(\cos \frac{\pi}{4}+\cos \frac{\pi}{12}\right) & \text { if } N=2 \\ \frac{1}{\sqrt{6}} & \text { if } N=3 \\ \frac{1}{(N-2) \sqrt{N}} & \text { if } N \geq 4\end{cases}
$$

\subsubsection{Eccentricity}

In this section, we associate to every subset $C \subseteq \mathbb{R}^{N}$ a parameter called eccentricity. Intuitively, eccentricity can be thought of as a measure of how much intersections of translated copies of $C$ centered at points in $Q$ deviate from the convex hull of $Q$. We recall that the Minkowski sum of two subsets $X \subset \mathbb{R}^{N}$ and $Y \subset \mathbb{R}^{N}$ is the subset defined by $X+Y=\{x+y \mid x \in X, y \in Y\}$. To simplify notations, we shall write $x+Y$ instead of $\{x\}+Y$. Let $B=\left\{x \in \mathbb{R}^{N}\right.$ $\|x\| \leq 1\}$ be the unit Euclidean ball centered at the origin. Given a non-negative real number $r$, we call the Minkowski sum $X+r B$ the Euclidean $r$-offset of $X$ and denote it by $X^{r}$. We write $\operatorname{Conv}(Q)$ for the convex hull of $Q \subset \mathbb{R}^{N}$. 
Definition 2. Given $\xi \geq 0$, we say that $C$ is $\xi$-eccentric if for all compact $Q \subset \mathbb{R}^{N}$, the following implication holds (see Figure 4):

$\bigcap_{q \in Q}(q+C) \neq \emptyset \Longrightarrow\left(\bigcap_{q \in Q}(q+C)\right) \cap(\operatorname{Conv}(Q)+\xi C) \neq \emptyset$.

The eccentricity of $C$ is the infimum of $\xi \geq 0$ such that $C$ is $\xi$-eccentric.
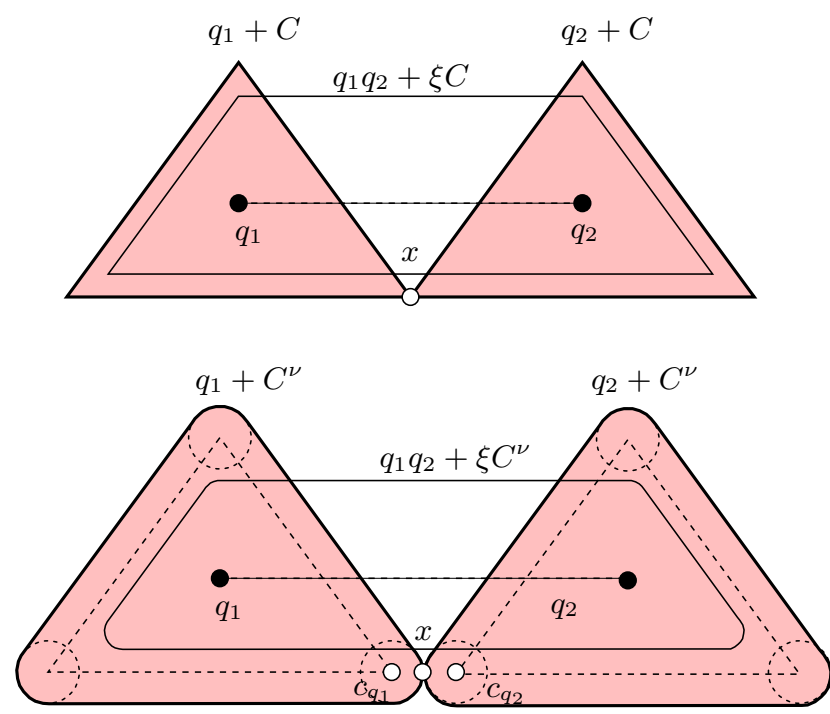

Figure 4: Top: two translated copies of a triangle $C$. The intersection point $x$ does not belong to any set $\operatorname{Conv}\left(\left\{q_{1}, q_{2}\right\}\right)+\xi C$ for $\xi<1$, thus showing that the eccentricity is 1 . Bottom: bulging the triangle makes its eccentricity drops to a value smaller than 1 .

Note that the eccentricity is a real number between 0 and 1. If the eccentricity of a compact set $C$ is $\xi$, then $C$ is $\xi$ eccentric. The property to be $\xi$-eccentric is invariant under bijective linear transformations. We now give eccentricities for simple objects and adopt the convention that all objects we consider in this paragraph have their centroids at the origin. Computations can be found in the technical report [5]. The eccentricity of the unit $N$-dimensional Euclidean ball $B$ is zero. More generally, $i$-dimensional Euclidean balls for $0 \leq i \leq N$ have eccentricity 0 . Ellipsoids which can be obtained from Euclidean balls by applying a linear transformation also have eccentricity 0. Symmetric compact convex sets in the plane have eccentricity 0 as well. At the opposite end of the spectrum, triangles have eccentricity 1 (see Figure [4]. In [5], we show that $N$-dimensional cubes have eccentricity $1-\frac{2}{N}$, for $N \geq 2$.

\subsection{Reconstruction Theorems}

First, we formulate a sampling condition inspired by the work in $2,3,10$ :

Definition 3. Given a non-negative real number $\varepsilon$ and a subset $C \subset \mathbb{R}^{N}$, we say that $P \subset \mathbb{R}^{N}$ is an $(\varepsilon, C)$-sample of $A \subset \mathbb{R}^{N}$ if $A \subset P+\varepsilon C$ and $P \subset A+\varepsilon B$.

Notice that $P$ is an $(\varepsilon, B)$-sample of $A$ if and only if the Hausdorff distance between $P$ and $A$ does not exceed $\varepsilon$. If
$B \subset C$, then an $(\varepsilon, B)$-sample is also an $(\varepsilon, C)$-sample. The reason why our definition is not symmetric with respect to $A$ and $P$ is to enhance conditions that are used in the proofs of our reconstruction theorems. Given a compact subset $A$ of $\mathbb{R}^{N}$, we recall that the medial axis $M$ of $A$ is the set of points in $\mathbb{R}^{N}$ which have at least two closest points in $A$. The reach of $A$ is the infimum of distances between points in $A$ and points in $M$ :

$$
\operatorname{reach}(A)=\inf _{a \in A, x \in M}\|a-x\| .
$$

Shapes with a positive reach include, but are not limited to, compact smooth surfaces with bounded curvatures. Intuitively, a shape with a positive reach cannot have sharp concave edges. Suppose $A$ has a positive reach. Given a convex set $C$ and an $(\varepsilon, C)$-sample $P$ of $A$, we would like to know whether the Minkowski sum $P+r C$ retrieves the topology of $A$. Theorem 1 answers the question when $P=A^{\varepsilon}$ is a Euclidean $\varepsilon$-offset of $A$ and Theorem 2 provides an answer when $P$ is a finite sample of $A$. Before stating our results, we start with an example which illustrates that not all convex sets $C$ can be used to reconstruct the topology of shapes with a positive reach.

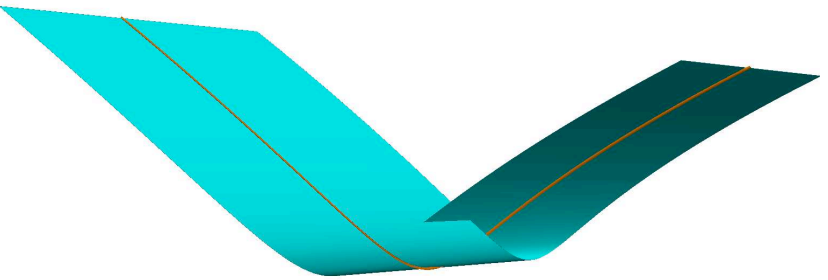

Figure 5: Cycle in the Minkowski sum of the moment curve with a segment.

Specifically, we take $A$ to be the moment curve $A=$ $\left\{\left(x_{1}, x_{2}, x_{3}\right) \in \mathbb{R}^{3} \mid x_{2}=x_{1}^{2}, x_{3}=x_{1}^{3}\right\}$ and prove that for $r$ arbitrarily small, we can always find a convex set $C$ such that the Minkowski sum $A+r C$ is not homotopy equivalent to $A$. For this, let $C$ be a segment of length 2 centered at the origin and contained in the straight-line $L_{t}=\left\{\left(x_{1}, x_{2}, x_{3}\right) \in \mathbb{R}^{3} \mid x_{2}=0, x_{3}=t^{2} x_{1}\right\}$ for some positive real number $t$. Observe that translated copies of $L_{t}$ intersect the moment curve $A$ in at most one point, except when the translated copy meets $A$ in the two points $a_{t}=\left(t, t^{2}, t^{3}\right)$ and $b_{t}=\left(-t, t^{2},-t^{3}\right)$. Hence, as $r$ increases, the Minkowski sum $A+r C$ undergoes a topology change when the two segments $a_{t}+r C$ and $b_{t}+r C$ meet (see Figure 5). This happens for $r=\left\|a_{t}-b_{t}\right\| / 2$ which can be made arbitrarily small by choosing $t$ small enough. We now list conditions we shall need on the compact convex set $C$, the last condition being only for the second theorem:

(i) $B \subset C \subset \delta B$ for some $\delta \geq 1$;

(ii) $C$ is $(\theta, \varkappa)$-round for $\theta \leq \theta_{N}=\arccos \left(-\frac{1}{N}\right)$ and $\varkappa>0$;

(iii) $C$ is $\xi$-eccentric for $\xi<1$.

The two conditions (ii) and (iii) do not imply each other. Indeed, any equilateral triangle in $\mathbb{R}^{2}$ with centroid the origin has eccentricity 1 and a positive $\theta$-roundness for all $0<\theta \leq \theta_{2}$. Hence, it satisfies (ii) for some $\theta \leq \theta_{2}$ and some $\varkappa>0$ but not (iii) for any $\xi<1$. Conversely, a segment in $\mathbb{R}^{2}$ has $\theta_{2}$-roundness 0 and eccentricity 0 . Hence, it 
satisfies (iii) for $\xi=0$ but not (ii) for any $\theta \leq \theta_{2}$ and $\varkappa>0$. Table 1 gathers for different compact convex sets $C$ values of $\delta, \theta, \varkappa$ and $\xi$ for which conditions (i), (ii) and (iii) hold. To state our theorems, let us introduce:

$$
R_{r}=R-\frac{r}{4}-\sqrt{\frac{r}{4}\left(2 R+\frac{r}{4}\right)},
$$

and note that $R_{r}$ tends to $R$ as $\frac{r}{R} \rightarrow 0$.

THEOREM 1. Let $A$ be a compact subset of $\mathbb{R}^{N}$ with positive reach $R$. Let $C$ be a compact convex set of $\mathbb{R}^{N}$ satisfying conditions (i) and (ii). Then, $A^{\varepsilon}+r C$ deformation retracts to $A$ for all real numbers $\varepsilon>0$ and $r>0$ such that $\varepsilon+(\delta-1) r<\min \left\{R-r, R_{r / \varkappa}\right\}$.

The proof of Theorem 1 is given in Section 3 . Theorem 1 will be useful in establishing our second theorem:

TheOREM 2. Let $A$ be a compact subset of $\mathbb{R}^{N}$ with positive reach $R$. Let $C$ be a compact convex set of $\mathbb{R}^{N}$ satisfying conditions (i), (ii) and (iii). Let $P$ be a finite $(\varepsilon, C)$-sample of $A$. Then, the inclusion $A \hookrightarrow P+r C$ is a homotopy equivalence for all real numbers $\varepsilon>0$ and $r>0$ such that (1) $(\delta-1) r<\min \left\{R-r, R_{r / \varkappa}\right\}$, (2) $\delta r<R-\varepsilon$, (3) $\delta\left(r+\alpha_{0}\right)<R$ and (4):

$2 R-\sqrt{(R-\varepsilon)^{2}-(\delta r)^{2}}-\sqrt{R^{2}-\delta^{2}\left(r+\alpha_{0}\right)^{2}}<(1-\xi) r-\varepsilon$, where $\alpha_{0}=\xi r+R-\sqrt{(R-\varepsilon)^{2}-(\delta r)^{2}}$.

The proof of Theorem 2 is given in Section 4 Notice that Theorem 2 requires that $\theta \leq \theta_{N}, \varkappa>0$ and $\xi<1$. If these three conditions are fulfilled, then by choosing $r=$ $\frac{4 \varepsilon}{1-\xi}$ and $\frac{\varepsilon}{R}$ small enough, all the assumptions of Theorem 2 are satisfied, implying that the inclusion $A \hookrightarrow P+r C$ is a homotopy equivalence. Given a fixed convex set $C$, Table 1 gives numerical approximations of the largest value of $\frac{\varepsilon}{R}$ for which assumptions of Theorem 2 hold.

To conclude this section, we prove that condition (iii) in Theorem 2 is necessary. In other words, if we take a compact convex set $C$ which satisfies conditions (i) and (ii) but whose eccentricity is 1 , it may happen that for some sample $P$, the Minkowski sum $P+r C$ does not recover the topology of $A$, no matter what value $r$ takes in the interval $[\varepsilon, R-\varepsilon]$. To construct such an example, consider a parabola $A$ in the plane with equation $y=x^{2}$ and a finite sample $P \subset A$ symmetric with respect to the $y$-coordinate axis. Furthermore, we let $C$ be the equilateral triangle with centroid the origin and vertices $(0,-2),(\sqrt{3}, 1)$ and $(-\sqrt{3}, 1)$. We note that with increasing value of $r$, holes appear in the Minkowski sum $P+r C$ each time two triangles scaled by $r$ meet at a common vertex on the $y$-axis (see Figure 6). We then adjust the height of the sample points in such a way that $P+\varepsilon C$ does not have the correct topology and as $r$ increases, a hole appears in $P+r C$ each time a hole gets destroyed, as illustrated in Figure 6

\section{PROOF OF THEOREM 1}

In this section, we prove Theorem 1 In other words, we prove that for $r$ and $\varepsilon$ small enough with respect to the reach of $A$, the Minkowski sum $A^{\varepsilon}+r C$ deformation retracts to $A$. Our strategy is as follows. We consider two positive real numbers $\varepsilon^{\prime}$ and $\varepsilon^{\prime \prime}$ such that there is chain of inclusions:

$$
A \subset A^{\varepsilon}+r C \subset A^{\varepsilon^{\prime}} \subset A^{\varepsilon^{\prime \prime}}+r C
$$

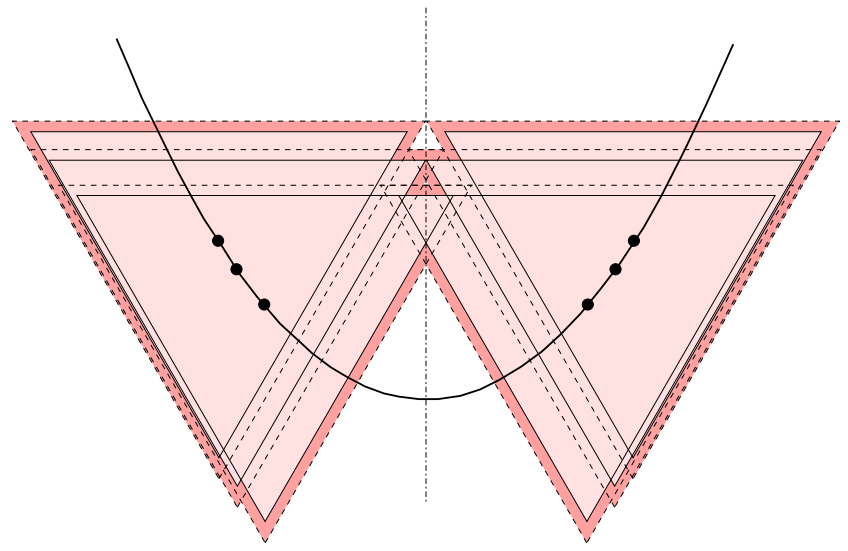

Figure 6: As the size of triangles increases, the hole created by the 4 upper points appears exactly when the hole created by the 4 lower points fills up.

and find conditions under which the third set deformation retracts to the first set and the fourth set deformation retracts to the second set. Applying the Sandwich Lemma allows us to conclude. All the difficulty comes from the second part of the proof which involves comparing the topology of two Euclidean offsets of $A+r C$, namely $A^{\varepsilon}+r C$ and $A^{\varepsilon^{\prime \prime}}+r C$. This leads us to study in details Euclidean offsets of Minkowski sums in Section 3.2 A powerful tool for detecting changes in the topology of Euclidean offsets consists in studying the critical points of distance functions. Key results concerning distance functions are recalled in Section 3.1

\subsection{Background on distance functions}

The distance function $d(\cdot, Y)$ to the compact subset $Y$ of $\mathbb{R}^{N}$ associates to each point $x \in \mathbb{R}^{N}$ its Euclidean distance to $Y, d(x, Y)=\min _{y \in Y}\|x-y\|$. The distance function $d(\cdot, Y)$ is 1-Lipschitz, but is not differentiable in general. Nonetheless, it is possible to define a notion of critical points analogue to the classical one for differentiable functions. Specifically, Grove defines in [17, page 360] critical points for the distance function to a closed subset of a Riemannian manifold. Using Equation (1.1)' in 17, page 360], we recast this definition in our context as follows. Let $\Gamma_{Y}(x)=\{y \in Y \mid d(x, Y)=\|x-y\|\}$ be the set of points in $Y$ closest to $x$ :

Definition 4. A point $x \in \mathbb{R}^{N}$ is a critical point of the distance function $d(\cdot, Y)$ if $x \in \operatorname{Conv}\left(\Gamma_{Y}(x)\right)$. The critical values of $d(\cdot, Y)$ are the images by $d(\cdot, Y)$ of its critical points.

Slightly recasting Proposition 1.8 in 17, page 362], we have:

Lemma 3 (Isotopy Lemma 17]). Let $0<\varepsilon \leq \varepsilon^{\prime}$. If the distance function $d(\cdot, Y)$ has no critical value in the interval $\left[\varepsilon, \varepsilon^{\prime}\right]$, then $Y^{\varepsilon}$ is a deformation retract of $Y^{\varepsilon^{\prime}}$.

If furthermore $Y$ has a positive reach $R$, then the projection map $\pi_{Y}$ which associates to each point $x \in Y^{\varepsilon}$ its closest point $\pi_{Y}(x)$ on $Y$ is well defined and continuous 16 . page 435]. Thus, the map $H:[0,1] \times Y^{\varepsilon} \rightarrow Y^{\varepsilon}$ defined by $H(t, x)=(1-t) x+t \pi_{Y}(x)$ is a deformation retraction of $Y^{\varepsilon}$ onto $Y$. It follows that: 
Table 1: Columns 2 to 5: values of $\delta, \theta, \varkappa$ and $\xi$ for which $B \subset C \subset \delta B$ and the convex set $C$ is $(\theta, \varkappa)-$ round and $\xi$-eccentric. Columns 6 and 7 : values of $R / \varepsilon$ and $r / \varepsilon$ for which Theorem 2 holds. Numerical values are obtained by brute force, enumerating all pairs $(\varepsilon, r)$ in a grid, checking if they satisfy conditions of Theorem 2 and keeping the one with largest $\varepsilon$.

\begin{tabular}{|c||c|c|c|c||c|c|}
\hline convex set $C$ & $\delta$ & $\theta$ & $\varkappa$ & $\xi$ & $R / \varepsilon$ & $r / \varepsilon$ \\
\hline \hline Euclidean ball $B \subset \mathbb{R}^{N}$ & 1 & 0 & 1 & 0 & 12.9781 & 3.95723 \\
\hline \hline cube $B_{\infty}$ in $\mathbb{R}^{N}$ & $\sqrt{N}$ & $\arccos \left(-\frac{1}{N}\right)$ & $\varkappa\left(B_{\infty}\right)$ & $1-\frac{2}{N}$ & & \\
\hline cube $B_{\infty}$ in $\mathbb{R}^{2}$ & $\sqrt{2}$ & $\frac{2 \pi}{3}$ & 0.65974 & 0 & 24.9973 & 4.04227 \\
\hline cube $B_{\infty}$ in $\mathbb{R}^{3}$ & $\sqrt{3}$ & $0.608 \pi$ & $\frac{1}{\sqrt{6}}$ & $1 / 3$ & 96.4687 & 6.14485 \\
\hline cube $B_{\infty}$ in $\mathbb{R}^{4}$ & $\sqrt{4}$ & $0.5804 \pi$ & $1 / 4$ & $1 / 2$ & 247.528 & 8.1826 \\
\hline cube $B_{\infty}$ in $\mathbb{R}^{5}$ & $\sqrt{5}$ & $0.5641 \pi$ & 0.149071 & $3 / 5$ & 508.183 & 10.2006 \\
\hline cube $B_{\infty}$ in $\mathbb{R}^{10}$ & $\sqrt{10}$ & $0.5319 \pi$ & 0.03953 & $4 / 5$ & 4505.44 & 20.2264 \\
\hline cube $B_{\infty}$ in $\mathbb{R}^{100}$ & 10 & $0.503183 \pi$ & 0.0010204 & $49 / 50$ & 4948245 & 200.232 \\
\hline \hline$p$-gon $\mathcal{P}_{p}$ in $\mathbb{R}^{2}(p$ even) & $\frac{1}{\cos \frac{\pi}{p}}$ & $\frac{2 \pi}{3}$ & $\varkappa\left(\mathcal{P}_{p}\right)$ & 0 & & \\
\hline \hline square in $\mathbb{R}^{2}$ & $\sqrt{2}$ & $\frac{2 \pi}{3}$ & 0.65974 & 0 & 24.9973 & 4.04227 \\
\hline hexagon in $\mathbb{R}^{2}$ & 1.1547 & $\frac{2 \pi}{3}$ & 0.69936 & 0 & 16.9858 & 3.99837 \\
\hline octagon in $\mathbb{R}^{2}$ & 1.08239 & $\frac{2 \pi}{3}$ & 0.793353 & 0 & 15.04119 & 3.98101 \\
\hline dodecagon in $\mathbb{R}^{2}$ & 1.03528 & $\frac{2 \pi}{3}$ & 0.8660254 & 0 & 13.84148 & 3.968 \\
\hline 36 -gon in $\mathbb{R}^{2}$ & 1.00382 & $\frac{2 \pi}{3}$ & 0.951917 & 0 & 13.07011 & 3.95844 \\
\hline 360 -gon in $\mathbb{R}^{2}$ & 1.00004 & $\frac{2 \pi}{3}$ & 0.9949868 & 0 & 12.97897 & 3.95724 \\
\hline
\end{tabular}

LEMMA 4. If $Y$ has a positive reach $R$, then $Y^{\varepsilon}$ deformation retracts to $Y$, for all $0 \leq \varepsilon<R$.

\subsection{Distance functions to Minkowski sums}

In what follows, $A$ designates a compact subset of $\mathbb{R}^{N}$ with positive reach $R$ and $C$ designates a compact convex set of $\mathbb{R}^{N}$. We begin with a technical lemma which will help us to situate critical points of the distance function to $A+C$, assuming $C$ is round enough.

Lemma 5. Consider a point $x \in \mathbb{R}^{N}$ such that $d(x, A+$ $C)<R$. Let $y_{1}, y_{2} \in \Gamma_{A+C}(x)$ be two points on $A+C$ with minimum distance to $x$. Suppose $C$ is $(\theta, \varkappa)$-round for $\varkappa>0$ and $\angle y_{1} x y_{2} \geq \theta$. Then, $d(x, A+C) \geq R_{1 / \varkappa}$.

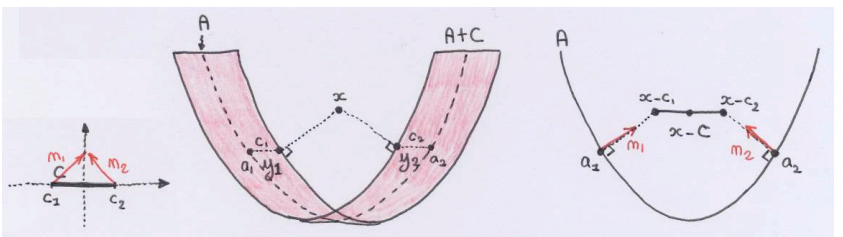

Figure 7: Notations for the proof of Lemma 5 .

Proof. Let $\rho=d(x, A+C)$. For $i \in\{1,2\}$, let $y_{i}=a_{i}+c_{i}$ with $a_{i} \in A$ and $c_{i} \in C$. Since $\rho=\left\|\left(x-c_{i}\right)-a_{i}\right\|<R$, it follows that $x-c_{i}$ has a unique projection $a_{i}=\pi_{A}\left(x-c_{i}\right)$ onto $A$ (see Figure 7). On the other hand, we know from 16. page 435] that the projection map $\pi_{A}$ onto $A$ is $\left(\frac{R}{R-\rho}\right)$ Lipschitz for points at distance less than $\rho$ from $A$. Thus,

$$
\left\|a_{1}-a_{2}\right\| \leq \frac{R}{R-\rho}\left\|c_{1}-c_{2}\right\| .
$$

Let $n_{i}=\frac{x-a_{i}-c_{i}}{\left\|x-a_{i}-c_{i}\right\|}$. Squaring both sides of the above inequality and plugging $a_{2}-a_{1}=c_{1}-c_{2}+\rho\left(n_{1}-n_{2}\right)$ into the left side, we obtain

$$
2\left(c_{1}-c_{2}\right) \cdot\left(n_{1}-n_{2}\right)+\rho\left\|n_{1}-n_{2}\right\|^{2} \leq \frac{2 R-\rho}{(R-\rho)^{2}}\left\|c_{1}-c_{2}\right\|^{2} .
$$

For $i \in\{1,2\}$, the unit vector $n_{i}$ belongs to $\mathcal{N}\left(c_{i}\right)$. Since $C$ is $(\theta, \varkappa)$-round and $\angle\left(n_{1}, n_{2}\right) \geq \theta$, it follows that $\left(c_{1}-c_{2}\right)$. $\left(n_{1}-n_{2}\right) \geq \varkappa\left\|c_{1}-c_{2}\right\|^{2}$ and

$$
\rho\left\|n_{1}-n_{2}\right\|^{2} \leq\left(\frac{2 R-\rho}{(R-\rho)^{2}}-2 \varkappa\right)\left\|c_{1}-c_{2}\right\|^{2} .
$$

In particular, this implies that $2 \varkappa \leq \frac{2 R-\rho}{(R-\rho)^{2}}$ or equivalently $\rho^{2}-2\left(R-\frac{1}{4 \varkappa}\right) \rho+R^{2}-\frac{R}{\varkappa} \leq 0$. Solving this quadratic inequality yields to the result.

As a consequence of the lemma above, if $x$ is sufficiently close to $A+C$, then the angle between any two vectors connecting $x$ to points in $\Gamma_{A+C}(x)$ is small which implies, in turn, that $x$ is not a critical point of $d(\cdot, A+C)$. The following lemma makes this idea precise.

Lemma 6. If $C$ is $(\theta, \varkappa)$-round with $\theta \leq \arccos \left(-\frac{1}{N}\right)$ and $\varkappa>0$, then the distance function $d(\cdot, A+C)$ has no critical value in the interval $\left(0, R_{1 / \varkappa}\right)$.

In order to prove Lemma 6, we need the following result also known as Jung's Theorem. Given a compact subset $K \subset \mathbb{R}^{N}$, we denote by $\operatorname{diam}(K)=\max _{p, q \in K} d(p, q)$ the diameter of $K$.

Lemma 7 (Jung's Theorem). The smallest ball enclosing a compact subset $K$ of $\mathbb{R}^{N}$ has radius

$$
r \leq \operatorname{diam}(K) \sqrt{\frac{N}{2(N+1)}} .
$$

Equality is attained for the regular $N$-simplex.

For a short proof of Jung's theorem, see 13. 
Proof of Lemma 6. Let $x \in \mathbb{R}^{N}$ and $\rho=d(x, A+C)$. Suppose $0<\rho<R_{1 / \varkappa}$ and let us prove that $x$ is noncritical. By Lemma 5 for all points $y_{1}, y_{2} \in \Gamma_{A+C}(x)$, we have $\angle y_{1} x y_{2}<\theta$. It follows that $\operatorname{diam}\left(\Gamma_{A+C}(x)\right)<2 \rho \sin \frac{\theta}{2}$. Using

$$
\sin \frac{\theta}{2}=\sqrt{\frac{1-\cos \theta}{2}} \leq \sqrt{\frac{N+1}{2 N}},
$$

and applying Jung's Theorem, we get that the smallest ball $B$ enclosing $\Gamma_{A+C}(x)$ has radius $r<\rho$. Let $S$ denote the sphere centered at $x$ with radius $\rho$. Observe that $\Gamma_{A+C}(x) \subset$ $S \cap B$. Since the radius of $B$ is smaller than $\rho$, the radical hyperplane $\Pi$ of the two spheres $S$ and $\partial B$ separates $x$ from $\Gamma_{A+C}(x)$. Thus $x \notin \operatorname{Conv}\left(\Gamma_{A+C}(x)\right)$ and $x$ is noncritical.

Combining Lemma 3 and Lemma 6 and using the fact that if $C$ is $(\theta, \varkappa)$-round, then $r C$ is $\left(\theta, \frac{\varkappa}{r}\right)$-round, we get immediately conditions under which a Euclidean offset of $A+r C$ deformation retracts to another Euclidean offset:

LEMMA 8. If $C$ is $(\theta, \varkappa)$-round with $\theta \leq \arccos \left(-\frac{1}{N}\right)$ and $\varkappa>0$, then $A^{\varepsilon}+r C$ is a deformation retract of $A^{\varepsilon^{\prime \prime}}+r C$ for all positive real numbers $r, \varepsilon$ and $\varepsilon^{\prime \prime}$ such that $\varepsilon \leq \varepsilon^{\prime \prime}<$ $R_{r / \varkappa}$.

We are now ready to establish the proof of our first reconstruction theorem.

Proof of Theorem 1, Equation (1) holds whenever $\varepsilon^{\prime}=$ $\varepsilon+\delta r$ and $\varepsilon^{\prime \prime}=\varepsilon+(\delta-1) r$. Since by hypothesis $\varepsilon \leq \varepsilon^{\prime \prime}<$ $R_{r / \varkappa}$, Lemma 8 implies that $A^{\varepsilon^{\prime \prime}}+r C$ deformation retracts to $A^{\varepsilon}+r C$. By hypothesis, we have $\varepsilon^{\prime}<R$ and therefore, $A^{\varepsilon^{\prime}}$ deformation retracts to $A$ from Lemma 4 Applying the Sandwich Lemma allows us to conclude.

\section{PROOF OF THEOREM 2}

In this section, we present our proof of Theorem 2, $A$ designates a compact subset of $\mathbb{R}^{N}$ whose reach $R$ is positive, $C$ is a compact convex set of $\mathbb{R}^{N}$ satisfying conditions (i), (ii) and (iii) and $P$ is a finite $(\varepsilon, C)$-sample of $A$. First, we introduce a set which will play a key role. Given three positive real numbers $\alpha, \beta$ and $r$, we set $\mathcal{A}_{p}(\alpha)=(p+r C) \cap$ $\left(A^{\beta}+\alpha C\right)$ and define

$$
\mathcal{H}(\alpha)=\bigcup_{p \in P} \operatorname{Conv} \mathcal{A}_{p}(\alpha)
$$

Our proof uses two carefully chosen positive constants $\alpha_{0}$ and $\alpha_{1}$ such that for all sufficiently small $\beta$, we have the sequence of inclusions (see Figure 8):

$$
A \subset \mathcal{H}\left(\alpha_{0}\right) \subset A^{\beta}+\alpha_{1} C \subset P+r C
$$

Having established this sequence of inclusions in Section 4.1. we find in Section 4.2 conditions under which $\mathcal{H}\left(\alpha_{0}\right) \hookrightarrow$ $P+r C$ is a homotopy equivalence. Combined with the conditions we found in Section 3 which ensure that $A \hookrightarrow$ $A^{\beta}+\alpha_{1} C$ is a homotopy equivalence, we deduce immediately using Lemma 1 (Sandwich Lemma) conditions under which $A \hookrightarrow P+r C$ is a homotopy equivalence.

\subsection{Establishing a key sequence of inclusions}

In this section, we find conditions under which inclusions in (2) hold. To establish the middle inclusion, we need the following key inclusion, illustrated in Figure 8

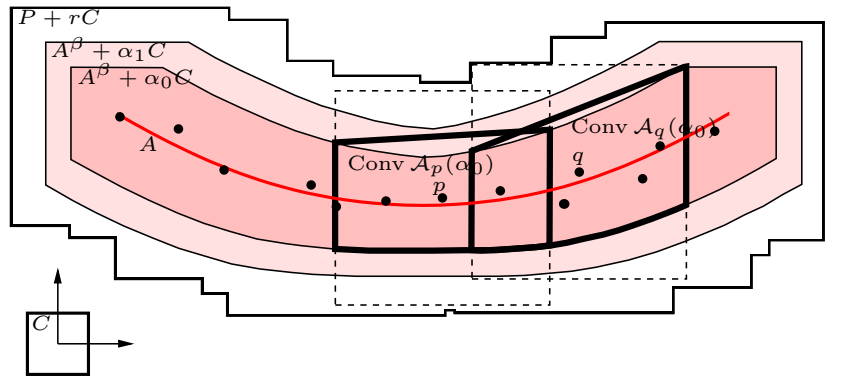

Figure 8: Nested sequence of objects considered for the proof of Theorem 2, Constants $\alpha_{0}$ and $\alpha_{1}$ are chosen such that $\operatorname{Conv} \mathcal{A}_{p}\left(\alpha_{0}\right)$ is contained in $A+\alpha_{1} C$ for all $p \in P$.

Lemma 9. Suppose $\delta\left(r+\alpha_{0}\right)<R-\beta$ and $\alpha_{1}-\alpha_{0} \geq$ $R-\sqrt{(R-\beta)^{2}-\delta^{2}\left(r+\alpha_{0}\right)^{2}}$. Then,

$$
\operatorname{Conv} \mathcal{A}_{p}\left(\alpha_{0}\right) \subset A+\alpha_{1} C .
$$

Proof. Let $A^{\prime}=A^{\beta} \cap\left(p+r C+\alpha_{0}(-C)\right)$. Note that $\mathcal{A}_{p}\left(\alpha_{0}\right) \subset A^{\prime}+\alpha_{0} C$ for if $x$ belongs to $\mathcal{A}_{p}\left(\alpha_{0}\right)=(p+r C) \cap$ $\left(A^{\beta}+\alpha_{0} C\right)$, we can find $c_{0}, c_{1} \in C$ and $a^{\prime} \in A^{\beta}$ such that $x=a^{\prime}+\alpha_{0} c_{0}=p+r c_{1}$, showing that $a^{\prime} \in A^{\prime}$ and $x \in$ $A^{\prime}+\alpha_{0} C$. Thus and using lemma 15 .

$\operatorname{Conv} \mathcal{A}_{p}\left(\alpha_{0}\right) \subset \operatorname{Conv}\left(A^{\prime}+\alpha_{0} C\right)=\operatorname{Conv}\left(A^{\prime}\right)+\alpha_{0} C$.

By construction, $A^{\prime}$ is contained in a ball of radius $\delta\left(r+\alpha_{0}\right)$. Applying Lemma 14 with $Q=A^{\prime}, \varepsilon=\beta$ and $\rho=\delta(2 r-\varepsilon)$, we get

$$
\operatorname{Conv}\left(A^{\prime}\right) \subset A+\left(R-\sqrt{(R-\beta)^{2}-\rho^{2}}\right) B
$$

if $\rho<R-\beta$. Thus, for all $p \in P$ we have $\operatorname{Conv} \mathcal{A}_{p}\left(\alpha_{0}\right) \subset$ $A+\alpha_{1} C$ whenever $\delta\left(r+\alpha_{0}\right)<R-\beta$ and $\alpha_{1}-\alpha_{0} \geq R-$ $\sqrt{(R-\beta)^{2}-\delta^{2}\left(r+\alpha_{0}\right)^{2}}$.

Taking the union over all points $p \in P$ on both sides of the inclusion in Lemma 9 we get immediately the middle inclusion in $(2)$, i.e. $\mathcal{H}\left(\alpha_{0}\right) \subset A^{\beta}+\alpha_{1} C$. The left-most and right-most inclusions in (2) are easy to establish, using $A \subset P+\varepsilon C$ and $B \subset C$.

Lemma 10. The sequence of inclusions in (2) holds whenever $\alpha_{1} \leq r-\varepsilon-\beta, \delta\left(r+\alpha_{0}\right)<R-\beta$ and $\alpha_{1}-\alpha_{0} \geq$ $R-\sqrt{(R-\beta)^{2}-\delta^{2}\left(r+\alpha_{0}\right)^{2}}$.

\subsection{A homotopy equivalence for nested collec- tions of convex sets}

It is not difficult to see that the inclusion $\mathcal{H}(\alpha) \subset P+r C$ holds for all positive real numbers $\alpha$ and $\beta$. The goal of this section is to find conditions under which the inclusion map $\mathcal{H}(\alpha) \hookrightarrow P+r C$ is a homotopy equivalence. For this, we use covers of $\mathcal{H}(\alpha)$ and $P+r C$ by finite collections of convex sets. Specifically, we have $\mathcal{H}(\alpha)=\bigcup_{p \in P} \operatorname{Conv} \mathcal{A}_{p}(\alpha)$ and $P+r C=\bigcup_{p \in P}(p+r C)$. Since sets in the two collections $\left\{\text { Conv } \mathcal{A}_{p}(\alpha)\right\}_{p \in P}$ and $\{p+r C\}_{p \in P}$ are convex, we can apply Leray's theorem 20] to each, and obtain that the union of sets in each collection has the same homotopy type as its associated nerve:

$$
\begin{aligned}
\mathcal{H}(\alpha) & \simeq \operatorname{Nerve}\left\{\operatorname{Conv} \mathcal{A}_{p}(\alpha)\right\}_{p \in P} \\
P+r C & \simeq \operatorname{Nerve}\{p+r C\}_{p \in P}
\end{aligned}
$$


A key step consists in proving that, for suitable values of $\alpha$, the nerves of the two collections are actually the same. As a consequence, $\mathcal{H}(\alpha)$ and $P+r C$ have the same homotopy type. We strengthen this result thanks to Lemma 11 and state conditions under which the inclusion $\mathcal{H}(\alpha) \hookrightarrow P+r C$ is a homotopy equivalence in Lemma 12

Lemma 11. Consider two finite collections of compact convex sets of $\mathbb{R}^{N}, \mathcal{C}=\left\{C_{i}\right\}_{i \in I}$ and $\mathcal{D}=\left\{D_{i}\right\}_{i \in I}$ such that $C_{i} \subset D_{i}$ for all $i \in I$ and suppose the two collections have the same nerve. Then, the inclusion $\bigcup_{i} C_{i} \hookrightarrow \bigcup_{i} D_{i}$ is a homotopy equivalence.

From Corollary 4G.3 in [18 also known as Leray's theorem 20 or the Nerve Lemma, it is clear that $\bigcup_{i} C_{i}$ and $\bigcup_{i} D_{i}$ which share the same nerve have the same homotopy type. But, we need here a stronger result, namely that the inclusion $\bigcup_{i} C_{i} \hookrightarrow \bigcup_{i} D_{i}$ is a homotopy equivalence. Even though this fact can be deduced from a result in 7], we provide below a short proof to make the paper self-contained.

Proof. Let $K(\mathcal{C})$ be the abstract simplicial complex whose simplices are the (non-empty) subsets of indices $\sigma \subset I$ such that $\bigcap_{i \in \sigma} C_{i} \neq \emptyset$. Since the two collections $\mathcal{C}$ and $\mathcal{D}$ have the same nerves, $K(\mathcal{C})=K(\mathcal{D})$ and we let $K=K(\mathcal{C})$.

For every subset of indices $\sigma \notin K$, a standard compactness argument yields a real number $\rho_{\sigma}>0$ such that $\bigcap_{i \in \sigma} D_{i}^{\rho_{\sigma}}=$ $\emptyset$. Let $\rho=\min _{\sigma \notin K} \rho_{\sigma}$ and define the open set $O_{i}=\{x \in$ $\left.\mathbb{R}^{N}, d\left(x, D_{i}\right)<\rho\right\}$ for every $i \in I$. By construction, the nerve of the collection $\mathcal{O}=\left\{O_{i}\right\}_{i \in I}$ is the same as the nerve of $\mathcal{D}$ and $K(\mathcal{O})=K$. For each $\sigma \in K$, we introduce the possibly empty open set:

$$
U_{\sigma}=\bigcap_{i \in \sigma} O_{i} \backslash \bigcup_{i \notin \sigma} D_{i}
$$

It is obvious from the definition that $\bigcup_{\sigma \in K} U_{\sigma} \subset \bigcup_{i \in I} O_{i}$. Let us associate to each point $x \in \bigcup_{i \in I} O_{i}$ the subset of indices $\tau(x)=\left\{i \in I, x \in O_{i}\right\}$. Since $x \in U_{\tau(x)}$, it follows that:

$$
\bigcup_{\sigma \in K} U_{\sigma}=\bigcup_{i \in I} O_{i}
$$

Let us consider a partition of unity $\left\{\phi_{\sigma}\right\}_{\sigma \in K}$ subordinate to the open cover $\left\{U_{\sigma}\right\}_{\sigma \in K}[23$, page 22]. Note that the map $\phi_{\sigma}$ is identically zero for the simplices $\sigma$ for which $U_{\sigma}=\emptyset$. For each simplex $\sigma \in K$, we choose an arbitrary point $c_{\sigma} \in$ $\bigcap_{i \in \sigma} C_{i}$ and introduce the map $h: \bigcup_{i \in I} D_{i} \rightarrow \mathbb{R}^{N}$ defined by:

$$
h(x)=\sum_{\sigma \in K} \phi_{\sigma}(x) c_{\sigma}
$$

By construction, $h$ is continuous. We claim that $x \in D_{i} \Longrightarrow$ $h(x) \in C_{i}$. Indeed, if $x \in D_{i}$ and $\phi_{\sigma}(x) \neq 0$, one has $i \in \sigma$ and therefore $c_{\sigma} \in C_{i}$. Hence, the non-zero terms in the above sum is a convex combination of points in $C_{i}$ and the claim follows from the convexity of $C_{i}$. Let us prove that $h$ is a homotopy inverse of the inclusion map $g: \bigcup_{i} C_{i} \rightarrow \bigcup_{i} D_{i}$. In other words, we have to check that $g \circ h$ is homotopic to the identity of $\bigcup_{i} D_{i}$ and $h \circ g$ is homotopic to the identity of $\bigcup_{i} C_{i}$. This can be done using twice the homotopy $H(x, t)=$ $(1-t) \cdot x+t \cdot h(x)$, first considered as a map from $\bigcup_{i} D_{i} \times[0,1]$ into $\bigcup_{i} D_{i}$, second considered as a map from $\bigcup_{i} C_{i} \times[0,1]$ into $\bigcup_{i}^{i} C_{i}$.
Lemma 12. Consider positive real numbers $r, \varepsilon, \alpha$ and $\beta$ such that $\delta r<R-\varepsilon, \delta(r+\alpha)<R-\beta$ and $\alpha \geq \xi r+R-$ $\sqrt{(R-\varepsilon)^{2}-(\delta r)^{2}}$. Then, the inclusion $\mathcal{H}(\alpha) \hookrightarrow P+r C$ is a homotopy equivalence.

Proof. We prove the lemma in three stages:

(a) First, we prove that for $\delta r<R-\varepsilon$ and $\alpha \geq \xi r+R-$ $\sqrt{(R-\varepsilon)^{2}-(\delta r)^{2}}$, we have

$$
\text { Nerve }\{p+r C\}_{p \in P}=\operatorname{Nerve}\left\{\mathcal{A}_{p}(\alpha)\right\}_{p \in P} .
$$

Note that this is equivalent to proving that for all subsets $Q \subset P$,

$$
\bigcap_{q \in Q}(q+r C) \neq \emptyset \Longleftrightarrow \bigcap_{q \in Q}\left[(q+r C) \cap\left(A^{\beta}+\alpha C\right)\right] \neq \emptyset .
$$

One direction is trivial: if a point belongs to the intersection on the right, then it belongs to the intersection on the left. Suppose now that $\bigcap_{q \in Q}(q+r C) \neq \emptyset$. In particular, using $C \subset \delta B$ this means that $Q$ can be enclosed in a ball of radius $\rho=\delta r$. Since $C$ is $\xi$-eccentric, there exists $z \in \bigcap_{q \in Q}(q+r C)$ such that $z \in \operatorname{Conv}(Q)+\xi r C$. Since $P$ is an $(\varepsilon, C)$-sample of $A$, we have $Q \subset P \subset A^{\varepsilon}$. Applying Lemma 14, we get that $\operatorname{Conv}(Q) \subset A^{\alpha-\xi r}$. Hence and using $B \subset C$, we get $z \in A+(\alpha-\xi r) B+\xi r C \subset A^{\beta}+\alpha C$.

(b) Second, we prove that

$$
\text { Nerve }\{p+r C\}_{p \in P}=\operatorname{Nerve}\left\{\operatorname{Conv} \mathcal{A}_{p}(\alpha)\right\}_{p \in P} .
$$

From Lemma 9, we obtain the sequence of inclusions

$$
\mathcal{A}_{p}(\alpha) \subset \operatorname{Conv} \mathcal{A}_{p}(\alpha) \subset \mathcal{A}_{p}\left(\alpha^{\prime}\right),
$$

for $\delta(r+\alpha)<R-\beta$ and $\alpha^{\prime}=\alpha+R-\sqrt{(R-\beta)^{2}-\delta^{2}(r+\alpha)^{2}}$. Taking the intersection over all points $q \in Q$, we get

$$
\bigcap_{q \in Q} \mathcal{A}_{q}(\alpha) \subset \bigcap_{q \in Q} \operatorname{Conv} \mathcal{A}_{q}(\alpha) \subset \bigcap_{q \in Q} \mathcal{A}_{q}\left(\alpha^{\prime}\right),
$$

and consequently

$\operatorname{Nerve}\left\{\mathcal{A}_{p}(\alpha)\right\} \subset \operatorname{Nerve}\left\{\operatorname{Conv} \mathcal{A}_{p}(\alpha)\right\} \subset \operatorname{Nerve}\left\{\mathcal{A}_{p}\left(\alpha^{\prime}\right)\right\}$,

where $p$ ranges over $P$. By Equation (3), the two nerves on the left and on the right are equal to Nerve $\{p+r C\}_{p \in P}$, showing that Nerve $\left\{\operatorname{Conv} \mathcal{A}_{p}(\alpha)\right\}_{p \in P}=\operatorname{Nerve}\{p+r C\}_{p \in P}$. (c) Third, noticing that $\operatorname{Conv} \mathcal{A}_{p}(\alpha) \subset p+r C$ for all $p$, we apply Lemma 11 to the two collections of convex sets $\mathcal{C}=\left\{\operatorname{Conv} \mathcal{A}_{p}(\alpha)\right\}_{p \in P}$ and $\mathcal{D}=\{p+r C\}_{p \in P}$.

We conclude this section by the proof of our second reconstruction theorem.

Proof of Theorem 2, For $\beta$ small enough, we have $\beta+$ $(\delta-1) r<\min \left\{R-r, R_{r / \varkappa}\right\}, \delta\left(r+\alpha_{0}\right)<R-\beta$ and

$$
\begin{array}{r}
2 R-\sqrt{(R-\varepsilon)^{2}-(\delta r)^{2}}-\sqrt{(R-\beta)^{2}-\delta^{2}\left(r+\alpha_{0}\right)^{2}} \\
\leq(1-\xi) r-\varepsilon-\beta .
\end{array}
$$

Setting $\alpha_{1}=\alpha_{0}+R-\sqrt{(R-\beta)^{2}-\delta^{2}\left(r+\alpha_{0}\right)^{2}}$, the above inequality can be rewritten as $\alpha_{1} \leq r-\varepsilon-\beta$. Thus, the sequence of inclusions in (2) holds by Lemma 10 Furthermore, the inclusion $\mathcal{H}(\alpha) \hookrightarrow P+r C$ is a homotopy equivalence by Lemma 12. Since $\alpha_{1} \leq r$ and $\beta+(\delta-1) r<\min \left\{R-r, R_{r / \varkappa}\right\}$ imply $\beta+(\delta-1) \alpha_{1}<\min \left\{R-\alpha_{1}, R_{\alpha_{1} / \varkappa}\right\}$, the inclusion $A \hookrightarrow A^{\beta}+\alpha{ }_{1} C$ is a homotopy equivalence by Theorem 1 Applying the Sandwich Lemma allows us to conclude. 


\section{DISCUSSION}

In this paper, we have exhibited a class of compact convex sets whose Minkowski sum with a sufficiently dense sample retrieves the topology of the sampled shape. Compact convex sets in this class possess three properties: a non-empty interior, a positive $\theta_{N}$-roundness and an eccentricity smaller than 1. In particular, this class contains Euclidean balls but, more interestingly, also includes $N$-dimensional cubes, with potential algorithmic applications in high dimensions.

Results in this paper raise a number of questions. For instance, it would be interesting to know what is the lowest density of sample points Theorem 2 authorizes and if this number is tight, especially for $N$-dimensional balls. Also, Theorem 2 requires the sample $P$ to be finite and the shape $A$ to have a positive reach. Can we relax these two conditions?

\section{REFERENCES}

[1] N. Amenta, D. Attali, and O. Devillers. A tight bound for the Delaunay triangulation of points on a pol yhedron. Research Report 6522, INRIA, May 2008.

[2] N. Amenta and M. Bern. Surface reconstruction by Voronoi filtering. Discrete and Computational Geometry, 22(4):481-504, 1999.

[3] N. Amenta, S. Choi, T. Dey, and N. Leekha. A simple algorithm for homeomorphic Surface Reconstruction. International Journal of Computational Geometry and Applications, 12:125-141, 2002.

[4] N. Amenta, S. Choi, and R. Kolluri. The power crust, unions of balls, and the medial axis transform. Computational Geometry: Theory and Applications, 19(2-3):127-153, 2001.

[5] D. Attali and A. Lieutier. Reconstructing shapes with guarantees by unions of convex sets. Archive Hal, hal-00427035, 2009.

[6] D. Attali and A. Lieutier. Optimal reconstruction might be hard. In 26th Ann. Sympos. Comput. Geom., Snowbird, Utah, 2010.

[7] P. Bendich, D. Cohen-Steiner, H. Edelsbrunner, J. Harer, and D. Morozov. Inferring local homology from sampled stratified spaces. In Proc. 48th Ann. Sympos. Found. Comput. Sci., pages 536-546, 2007.

[8] J.-D. Boissonnat, O. Devillers, and S. Hornus. Incremental construction of the Delaunay triangulation and the Delaunay graph in medium dimension. In Proc. ACM Symposium on Computational Geometry, June 2009.

[9] P. Cannarsa and C. Sinestrari. Semiconcave functions, Hamilton-Jacobi equations, and optimal control. Birkhauser, 2004.

[10] F. Chazal, D. Cohen-Steiner, and A. Lieutier. A sampling theory for compact sets in Euclidean space. Discrete and Computational Geometry, 41(3):461-479, 2009.

[11] F. Chazal and A. Lieutier. The $\lambda$-medial axis. Graphical Models, 67(4):304-331, 2005.

[12] F. Chazal and A. Lieutier. Smooth Manifold Reconstruction from Noisy and Non Uniform Approximation with Guarantees. Computational Geometry: Theory and Applications, 40:156-170, 2008.
[13] V. de Silva and R. Ghrist. Coverage in sensor networks via persistent homology. Algebraic \& Geometric Topology, 7:339-358, 2007.

[14] T. Dey, J. Giesen, E. Ramos, and B. Sadri. Critical points of the distance to an epsilon-sampling of a surface and flow-complex-based surface reconstruction. In Proc. of the twenty-first annual symposium on Computational geometry, page 227. ACM, 2005.

[15] H. Edelsbrunner. The union of balls and its dual shape. Discrete Computational Geometry, 13(1):415-440, 1995.

[16] H. Federer. Curvature measures. Trans. Amer. Math. Soc, 93:418-491, 1959.

[17] K. Grove. Critical point theory for distance functions. In Proc. of Symposia in Pure Mathematics, volume 54, pages 357-386, 1993.

[18] A. Hatcher. Algebraic topology. Cambridge University Press, 2002.

[19] D. Koutroufiotis. On Blaschke's rolling theorems. Archiv des Mathematik, 23(1):655-660, 1972.

[20] J. Leray. Sur la forme des espaces topologiques et sur les points fixes des représentations. J. Math. Pures Appl., 24:95-167, 1945.

[21] J. R. Munkres. Topology. Prentice Hall, 2000.

[22] P. Niyogi, S. Smale, and S. Weinberger. Finding the Homology of Submanifolds with High Confidence from Random Samples. Discrete Computational Geometry, 39(1-3):419-441, 2008.

[23] L. Schwartz. Théorie des distributions. Hermann, Paris, 1966.

\section{APPENDIX}

\section{A. BASIC PROPERTIES}

In this appendix, we present basic properties relating the smallest ball enclosing $Q \subset \mathbb{R}^{N}$ and the convex hull of $Q$.

Lemma 13. Consider a subset $Q \subset \mathbb{R}^{N}$ whose smallest enclosing ball has radius $r$. Then, $\operatorname{Conv}(Q) \subset \bigcup_{q \in Q} B(q, r)$.

Proof. For all $q \in \operatorname{Conv}(Q)$, there are points $q_{1}, \ldots, q_{n}$ in $Q$ and non-negative real numbers $\alpha_{1}, \ldots, \alpha_{n}$ summing up to 1 , such that $q=\sum_{i=1}^{n} \alpha_{i} q_{i}$. Let $\pi_{i}(x)=\left\|x-q_{i}\right\|^{2}-r^{2}$ be the power distance of $x \in \mathbb{R}^{N}$ from $B_{i}=B\left(q_{i}, r\right)$ and note that $B_{i}=\pi_{i}^{-1}(-\infty, 0]$. Let $\pi(x)=\sum_{i=1}^{n} \alpha_{i} \pi_{i}(x)$ and set $B=\pi^{-1}(-\infty, 0]$. We prove that $\bigcap_{i=1}^{n} B_{i} \subset B \subset \bigcup_{i=1}^{n} B_{i}$. Indeed, if a point $x$ belongs to all balls $B_{i}$, then $\pi_{i}(x) \leq 0$ for all $1 \leq i \leq n$, which implies $\pi(x) \leq 0$. On the other hand, if $\pi(x) \leq 0$ then $\pi_{i}(x) \leq 0$ for at least one index $i$, which implies that $x$ belongs to at least one ball $B_{i}$. Now, our choice of $r$ as the radius of the smallest ball enclosing $Q$ implies that $\bigcap_{i=1}^{n} B_{i} \neq \emptyset$, showing that $B$ is non-empty. Thus, $B$ is a ball and it is not difficult to see that its center is point $q$. It follows that $q \in B \subset \bigcup_{i=1}^{n} B_{i}$, which concludes the proof.

The next lemma states that the convex hull of a set of points cannot be too far away from a shape with positive reach, assuming the set of points is close to the shape and are enclosed in a ball of small radius. Formally:

Lemma 14. Consider a subset $Q \subset A^{\varepsilon}$ in $\mathbb{R}^{N}$ and suppose $Q$ can be enclosed in a ball of radius $\rho<R-\varepsilon$. Then, $\operatorname{Conv}(Q) \subset A^{\alpha}$ for $\alpha \geq R-\sqrt{(R-\varepsilon)^{2}-\rho^{2}}$. 


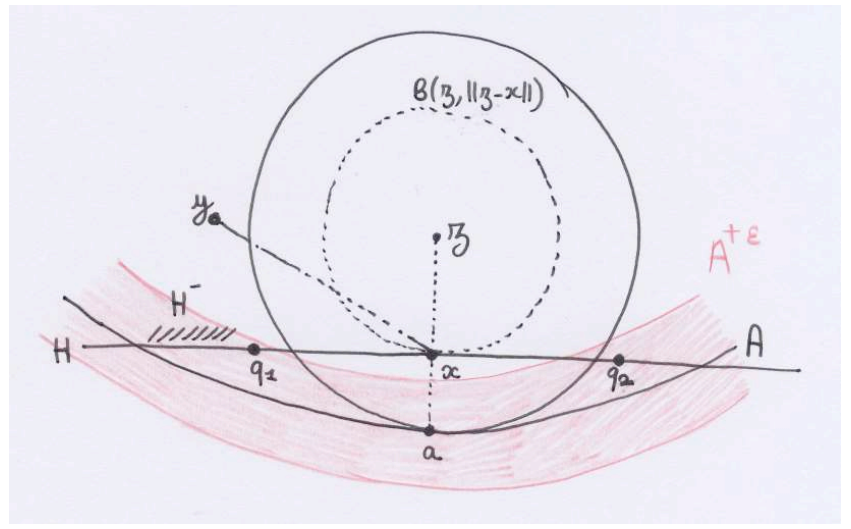

Figure 9: Notations for the proof of Lemma 14.

Proof. Suppose $R<+\infty$ for otherwise, $A$ is convex and $\operatorname{Conv}(Q) \subset A^{\varepsilon}$. Let $x$ be a point on $\operatorname{Conv}(Q)$ furthest away from $A$. By Lemma 13 there exists a point $q \in Q$ such that $\|x-q\| \leq \rho$. Thus, $d(x, A) \leq\|x-q\|+d(q, A) \leq \rho+\varepsilon<R$, showing that $x$ has a unique projection $a$ onto $A$. We claim that the plane $H$ passing through $x$ and orthogonal to the segment $x a$ is a supporting plane of the convex hull of $Q$. To prove this, consider the two open half-spaces that $H$ bounds and let $H^{-}$be the one half-space that does not contain $a$. Furthermore, consider the half-line with origin $a$ that passes through $x$ and let $z$ be the point on this half-line at distance $R$ from $a$ (see Figure 9). By construction, $B(z, R)$ is tangent to $A$ at $a$ and its interior does not intersect $A$. We prove that $\operatorname{Conv}(Q) \cap H^{-}=\emptyset$. Suppose for a contradiction that there exists a point $y \in \operatorname{Conv}(Q) \cap H^{-}$. Then, the whole segment $x y$ belongs to $\operatorname{Conv}(Q)$ and in particular intersects $B(z,\|z-x\|)$. But points in the interior of $B(z,\|z-x\|)$ are furthest away from $A$ than $x$, contradicting the definition of $x$ as the point of $\operatorname{Conv}(Q)$ furthest away from $A$. It follows that $H$ is a supporting plane of the convex hull of $Q$ as claimed. Thus, $Q \cap H$ is non-empty and can be enclosed in a ball of radius smaller or equal to $\rho$. The convex hull of $Q \cap H$ contains $x$ and by Lemma 13 there exists a point $q^{\prime} \in Q \cap H$ such that $\left\|x-q^{\prime}\right\| \leq \rho$. On the other hand, $\left\|z-q^{\prime}\right\| \geq R-\varepsilon$. It follows that $\|x-a\|=R-\sqrt{\left\|z-q^{\prime}\right\|^{2}-\left\|x-q^{\prime}\right\|^{2}} \leq$ $\alpha$.

Lemma 15. For any subset $Q \subset \mathbb{R}^{N}$ and any convex set $C \subset \mathbb{R}^{N}, \operatorname{Conv}(Q+C)=\operatorname{Conv}(Q)+C$.

The proof is straightforward and hence omitted.

\section{B. PROOF OF LEMMA 2}

We start with a preliminary lemma.

Lemma 16. For all points $c_{1}, c_{2} \in C, c_{1} \neq c_{2}$ and all vectors $n_{1} \in \mathcal{N}\left(c_{1}\right)$ and $n_{2} \in \mathcal{N}\left(c_{2}\right)$, we have

$$
\frac{\left(c_{1}-c_{2}\right) \cdot\left(n_{1}-n_{2}\right)}{\left\|c_{1}-c_{2}\right\|^{2}}=\frac{1}{2}\left(\kappa_{c_{1}, n_{1}}\left(c_{2}\right)+\kappa_{c_{2}, n_{2}}\left(c_{1}\right)\right),
$$

where $\kappa_{x, n}(y)$ is the curvature of the sphere passing through points $x$ and $y$ and with outer normal $n$ at point $x$.

Proof. See Figure 10, left. Let $S_{i}$ be the sphere passing through points $c_{1}$ and $c_{2}$ with outer normal $n_{i}$ at $c_{i}$, for $i \in\{1,2\}$. Let $n_{2}^{\prime}$ be the outer unit normal to $S_{1}$ at $c_{2}$ and $n_{1}^{\prime}$ be the outer unit normal to $S_{2}$ at $c_{1}$. We have

$$
\begin{aligned}
& \left(c_{1}-c_{2}\right) \cdot\left(n_{1}-n_{2}^{\prime}\right)=\kappa_{c_{1}, n_{1}}\left(c_{2}\right)\left\|c_{1}-c_{2}\right\|^{2} \\
& \left(c_{1}-c_{2}\right) \cdot\left(n_{1}^{\prime}-n_{2}\right)=\kappa_{c_{2}, n_{2}}\left(c_{1}\right)\left\|c_{1}-c_{2}\right\|^{2} \\
& \left(c_{1}-c_{2}\right) \cdot\left(n_{2}^{\prime}+n_{1}\right)=0 \\
& \left(c_{2}-c_{1}\right) \cdot\left(n_{1}^{\prime}+n_{2}\right)=0
\end{aligned}
$$

Summing up these four equations gives the result.

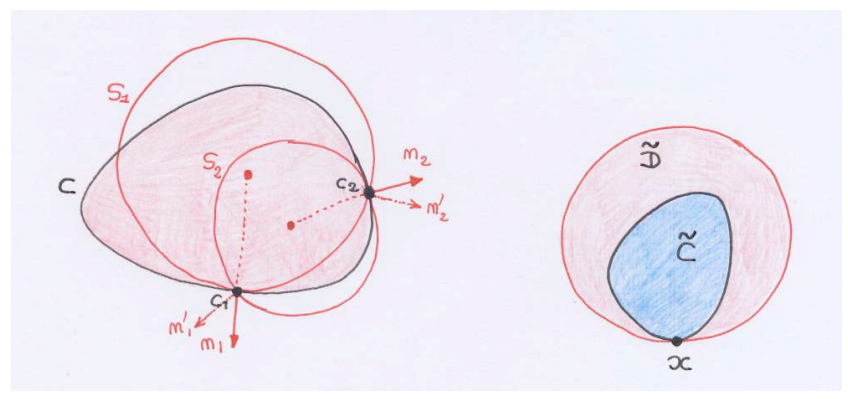

Figure 10: Notations for the proof of Lemma 16 and Lemma 2,

Proof of Lemma 2, We first prove that for all points $c_{1}, c_{2} \in \partial C$ with normals $n_{1}$ and $n_{2}$ respectively:

$$
\left(c_{1}-c_{2}\right) \cdot\left(n_{1}-n_{2}\right) \geq \kappa_{\min }(C)\left\|c_{1}-c_{2}\right\|^{2} .
$$

Consider a sphere $S$ tangent to $\partial C$ at point $x$ and meeting $\partial C$ in another point $y \neq x$. Let $D$ be the ball that $S$ bounds. We begin by proving that $\kappa_{\min }(D) \geq \kappa_{\min }(C)$. Consider a 2dimensional plane $P$ passing through $x$ and $y$ and containing the common normal to $\partial D$ and $\partial C$ at point $x$. In particular, $P$ passes through the center of $D$. We think of $\tilde{D}=D \cap P$ and $\tilde{C}=C \cap P$ as two compact convex sets in $\mathbb{R}^{2}$. By construction, $\partial \tilde{D}$ and $\partial \tilde{C}$ are $\mathrm{C}^{2}$-smooth curves tangent at point $x$ and meeting at point $y \neq x$. Thus, we reduced the geometric situation in $\mathbb{R}^{N}$ to the same situation in $\mathbb{R}^{2}$. Let us prove that $\kappa_{\min }(\tilde{D}) \geq \kappa_{\min }(\tilde{C})$. Suppose for a contradiction that $\kappa_{\min }(\tilde{D})<\kappa_{\min }(\tilde{C})$. In other words, the curvature of circle $\tilde{D}$ is smaller than the curvature at any point on the curve $\tilde{C}$. Theorem 1 in 19 tells us that $\tilde{C}$, except for $x$, lies in the interior of $\tilde{D}$, as illustrated in Figure 10 right. But, this contradicts the fact that $\tilde{C}$ intersects the boundary of $\tilde{D}$ in $y \neq x$. Thus, $\kappa_{\min }(\tilde{D}) \geq \kappa_{\min }(\tilde{C})$ and it follows that $\kappa_{\min }(D)=\kappa_{\min }(D \cap P) \geq \kappa_{\min }(C \cap P) \geq \kappa_{\min }(C)$, as claimed. In other words, given two points $x \neq y$ on the boundary of $C$ and a unit vector $n \in \mathcal{N}(x)$, we have just proved that the curvature $\kappa_{x, n}(y)$ of the sphere passing through $x$ and $y$ with outer normal $n$ at point $x$ satisfies $\kappa_{x, n}(y) \geq \kappa_{\min }(C)$. Applying Lemma 16 gives the claimed inequality.

To prove that the inequality is tight, note that if $c_{2}$ tends to $c_{1}$ along a curve $\gamma$ in $\partial C$, then the ratio

$$
\frac{\left(c_{1}-c_{2}\right) \cdot\left(n_{1}-n_{2}\right)}{\left\|c_{1}-c_{2}\right\|^{2}}
$$

tends to the absolute value of the normal curvature of $\gamma$ at $c_{1}$. In particular, if $\left|\kappa_{N-1}(x)\right|$ reaches its minimum at $x=c_{1}$ and the tangent line to $\gamma$ at $c_{1}$ is the associated principal direction, then the ratio tends to $\kappa_{\min }(C)$. 